\title{
IUE Monitoring of the Bright Seyfert 1 Galaxy Fairall 9
}

\author{
P. M. Rodríguez-Pascual ${ }^{1}$ \\ ESA-IUE Observatory, P.O. Box 50727, 28080 Madrid, Spain
}

\begin{abstract}
An eight-month spectrscopic monitoring campaign on the Seyfert 1 galaxy Fairall 9 has been carried out with the International $\mathrm{Ul}$ traviolet Explorer (IUE) in order to measure continuum-continuum and continuum-emission-line delays for a high-luminosity active galactic nucleus (AGN). The results are consistent with those of previous campaigns on lower luminosity AGNs: (i) there is no measurable time lag between different ultraviolet continuum bands, (ii) the measured time lags for the emission lines are relatively short, and (iii) the more rapid and stronger response to continuum changes of $\mathrm{He} I \mathrm{I}$, as compared to $\mathrm{C} I V$, is consistent with a stratified broad-line region (BLR) structure.
\end{abstract}

From 1994 April 28 to December 26, Fairall 9 was observed with the SWP camera $(1150-1950 \AA)$ on board the IUE satellite at nearly regular intervals of $\sim 4$ days. The observations were made in low-dispersion mode ( $\sim 6 \AA$ resolution) and resulted in 68 spectra corresponding to 63 different epochs.

The continuum light curve measured at $1390 \AA$ (observed wavelength) shows three 'events' of similar duration ( $\sim 70$ days), but with noticeably different amplitude (Fig. 1). The first two events show variations of up to $50 \%$, while during the last event the continuum varied by more than a factor of two. The Ly $\alpha$ light curve is qualitatively similar to that of the continuum, with two low-amplitude events $(\sim 15 \%)$ followed by a stronger $(\sim 35 \%)$ third one. Although still smaller than in the continuum, the amplitude of the He II variations is the largest among the emission lines. The detection of the first two events in the He II light curve, if at a level similar to those observed in Ly $\alpha$, is difficult because of the large uncertainties associated with individual $\mathrm{He}$ II measurements. The variations in the CIV line during this campaign $(\sim 20 \%)$ are much smaller than those in the continuum or the Ly $\alpha$ and He II emission lines.

The cross-correlation functions (CCFs) between the continuum and the strongest emission lines, computed using the linear interpolation method of Gaskell \& Sparke (1986) and the discrete correlation function (DCF) method of Edelson \& Krolik (1989), are shown in Fig. 1. The peak and the centroid of the CCFs for Ly $\alpha$ are 14 and 9 days respectively, while the corresponding values for HeIl are 11 and 6 days. The broad and weak peak of the CCF and DCF for C IV makes any estimate of a lag between this line and the continuum unreliable. However, the longer delay suggested in the CCF together with the much lower-amplitude variations are consistent with a stratified BLR.

\footnotetext{
${ }^{1}$ On behalf of the International AGN Watch consortium. The author is affiliated with the ESA Astrophysics Division, Space Science Department.
} 

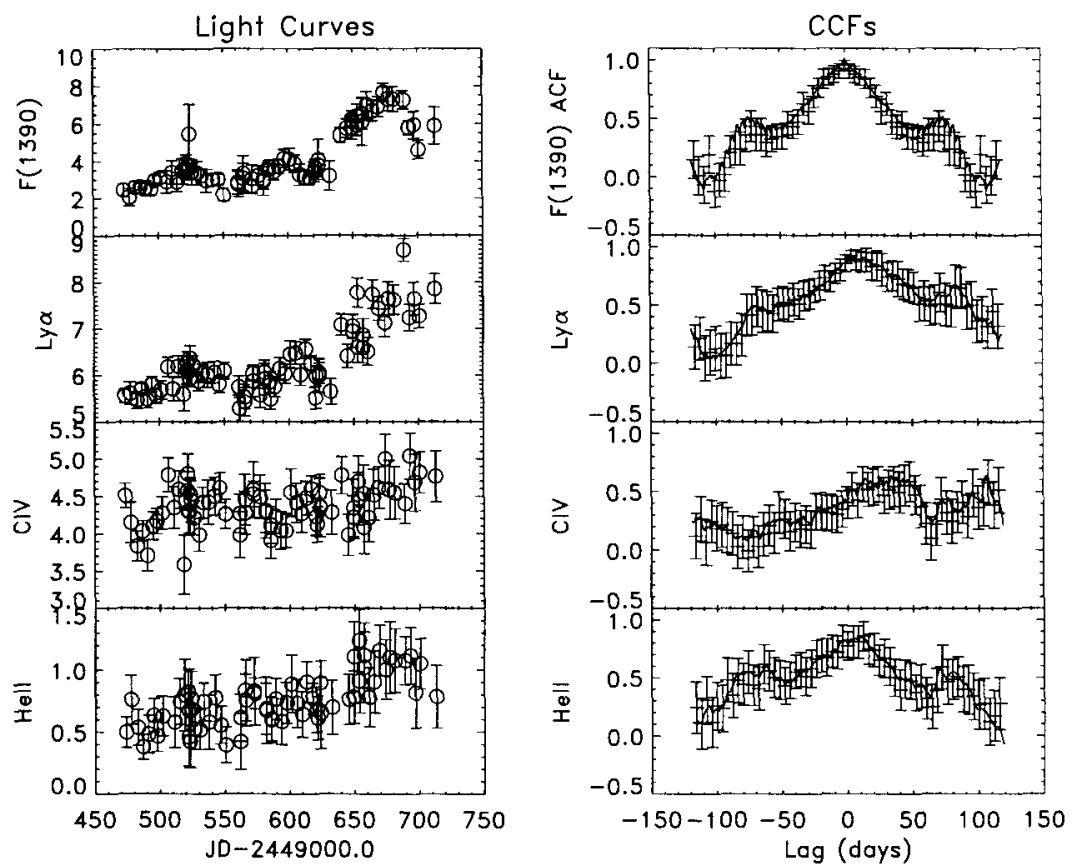

Figure 1. Left panel: Light curves of the continuum (in units of $10^{-14} \mathrm{ergs} \mathrm{s}^{-1} \mathrm{~cm}^{-2} \AA^{-1}$ ) and the strongest emission lines (in units of $10^{-12} \mathrm{ergs} \mathrm{s}^{-1} \mathrm{~cm}^{-2}$ ). Right panel: CCFs (solid lines) and DCFs (data points with error bars) between the continuum and the emission lines.

It is remarkable that the lags found for the Ly $\alpha$ and He II emission lines of Fairall 9 in this experiment are very similar to the lags found for the same lines of NGC 5548 during its monitoring campaign in 1989 (Clavel et al. 1991). Although the UV continuum luminosity of Fairall 9 during 1994 was close to its historical minimum, it was still 6 times higher than the UV continuum luminosity of NGC 5548 during 1989.

\section{References}

Clavel, J.. et al. 1989, ApJ, 366, 64 .

Edelson, R. A., \& Krolik, J. H. 1989, ApJ, 333, 646.

Gaskell, C. M., \& Sparke, L.S. 1986, ApJ, 305, 175. 